\title{
Technique for in situ measurement of free spectral range and transverse mode spacing of optical cavities
}

\author{
Alberto Stochino, ${ }^{1,2,3, *}$ Koji Arai, ${ }^{1}$ and Rana X. Adhikari ${ }^{1}$ \\ 'LIGO Laboratory, MS 100-36, California Institute of Technology, Pasadena, California 91125, USA \\ ${ }^{2}$ Università degli Studi di Siena, Dipartimento di Fisica, Via Roma 56, 53100 Siena, Italy \\ ${ }^{3}$ Centre for Gravitational Physics, Department of Quantum Science, The Australian National University, Canberra, \\ Australian Capital Territory 0200, Australia \\ *Corresponding author: stochino@ligo.caltech.edu
}

Received 14 June 2012; accepted 30 July 2012; posted 20 August 2012 (Doc. ID 170533); published 17 September 2012

\begin{abstract}
Length and $g$-factor are fundamental parameters that characterize optical cavities. We developed a technique to measure these parameters in situ by determining the frequency spacing between the resonances of fundamental and spatial modes of an optical cavity. Two laser beams are injected into the cavity, and their relative frequency is scanned by a phase-lock loop, while the cavity is locked to either laser. The measurement of the amplitude of their beat note in transmission reveals the resonances of the longitudinal and the transverse modes of the cavity and their spacing. This method proves particularly useful to characterize complex optical systems, including very long and/or coupled optical cavities, as in gravitational-wave interferometers. This technique and the results of its application to the coupled cavities of a $40 \mathrm{~m}$-long gravitational-wave interferometer prototype are presented here. (C) 2012 Optical Society of America
\end{abstract}

OCIS codes: $\quad 120.3180,120.2230,120.3940,350.1270$.

\section{Introduction}

The absolute length and mirror curvatures are defining parameters of an optical cavity. Together these quantities uniquely determine the free spectral range (FSR) and the transverse mode spacing (TMS), respectively the frequency spacing between different resonances of the cavity's longitudinal modes and the frequency spacing between the resonances of transverse and longitudinal modes.

FSR and TMS are usually accurately specified and it is thus very important to measure them with great precision. Over time, monitoring cavity length and mirror curvatures can track changes affecting the cavity geometry. For instance, variations of the cavity

$1559-128 \mathrm{X} / 12 / 276571-07 \$ 15.00 / 0$

(C) 2012 Optical Society of America length may reveal drifts of the mirrors' positions caused by ground displacement [1]; changes of the mirrors' curvatures can be evidence of deformations due to thermal effects [2]. Precise measurements of FSR and TMS can also allow the modeling of the cavity reflected and transmitted power by predicting the mode distribution around the cavity working point.

Measuring these parameters proves particularly challenging in long and complex optical cavities, such as those in gravitational-wave interferometers [3-6] . Precisely characterizing the optical cavities in these detectors is crucial to achieve their best sensitivity. Often three to five or more coupled optical cavities are controlled simultaneously through the sensing of RF phase-modulation sidebands added to the main laser. Resonance or antiresonance conditions of the sidebands in each part of the interferometer must be ensured at all times for optimal decoupling among 
the degrees of freedom [7]. When the RF modulation frequencies and the cavity lengths do not match, controlling the interferometer becomes more difficult. Also, a mismatch of more than a few centimeters in the arm cavities, or $\sim 1 \mathrm{~mm}$ in the recycling cavities, may reduce the sensitivity of the detector. Noise from the laser and the RF modulator may then enter the interferometer's auxiliary degrees of freedom and leak into the gravitational-wave channel by cross couplings [8-10].

Ye [11] showed that, in principle, subwavelength length measurement precision could be obtained by using a femtosecond laser. However this scheme is difficult to implement in situations where a dedicated ultrashort pulsed laser system may not bet readily available. Several different approaches have been tried in the past. In what was probably the simplest, Rakhmanov et al. [12] measured the length of a cavity with a precision of $4 \mathrm{~mm}$ by an optical vernier obtained by swinging the end mirror. In a later experiment, Rakhmanov et al. [2] measured the length of a $4 \mathrm{~km}$ cavity with $80 \mu \mathrm{m}$ precision by measuring the cavity's frequency response by frequency modulating the laser. In a similar way, Uehara and Ueda [13] measured the radius of curvature of the end mirror of a plano-concave cavity. Additionally, Araya et al. [1], following DeVoe and Brewer [14], estimated the length of a $300 \mathrm{~m}$ cavity with a relative precision of $10^{-9}$ by simultaneously locking to the cavity the laser's carrier and a phase-modulation sideband.

These techniques are difficult to scale and adapt to systems of very different lengths, or included in complex, coupled-cavity configurations. For example, frequency modulation techniques cannot be used in short gravitational-wave interferometers since any modulation of the laser at frequencies near the cavity FSR would be suppressed by the input mode-cleaner cavity. Tuning the sidebands' frequency as in Araya's technique would not be possible in systems including additional input cavities.

To circumvent these limitations, we developed an alternative interferometric technique to measure the FSR and TMS of optical cavities with a larger range of lengths or in complex optical setups. We then tested this technique on the optical cavities forming the LIGO $40 \mathrm{~m}$ gravitational-wave detector prototype at the California Institute of Technology. In the following, we describe this technique and present the results obtained in our experiments.

\section{Principles of the Technique}

The FSR of an optical cavity determines the frequency spacing $\nu_{\text {FSR }}$ between resonances of any given pair of cavity longitudinal modes. It is defined as [15]

$$
\nu_{\mathrm{FSR}}=\frac{c}{2 L},
$$

where $L$ is the cavity length and $c$ the speed of light. This definition can then be used to infer the cavity length from the direct measurement of the FSR. In a similar way, the mirrors' curvatures are inferred from the measurement of the TMS and the estimated cavity length.

Each Hermite-Gaussian mode is characterized by a different Guoy phase determining its specific resonant frequency in the cavity [15]. Because of this phase, the set of resonances of a generic $\mathrm{TEM}_{m n}$ mode is shifted from the resonances of the fundamental $\mathrm{TEM}_{00}$ mode by an integer multiple of the so-called transverse or spatial mode spacing. For a linear cavity, this is defined as

$$
\nu_{\mathrm{TMS}}=\nu_{\mathrm{FSR}} \frac{m+n}{\pi} \cos ^{-1} \sqrt{g_{1} g_{2}},
$$

where $g_{1}=\left(1-L / R_{1}\right)$ and $g_{2}=\left(1-L / R_{2}\right)$ are the g-parameters of the mirrors, with $R_{1}, R_{2}$ representing their respective radii of curvature, and $L$ the absolute length of the cavity. The product of the $g$-parameters $g_{1} g_{2}$ is often referred to as the cavity g-factor:

$$
g=g_{1} g_{2} .
$$

It follows from Eq. (2) that if the cavity mirrors are astigmatic, the resonances of complimentary modes, $\mathrm{TEM}_{m n}$ and $\mathrm{TEM}_{n m}$, are split. If we assume, for simplicity, that axes of the astigmatism for the two mirrors are aligned, different $g$-factors $g_{x}$ and $g_{y}$ can be associated with each of the two transverse spatial directions $x$ and $y$, respectively. (For more general astigmatic cavity cases, see [16].) In the case of astigmatic cavities, the definition of TMS is then generalized as

$$
\begin{aligned}
\nu_{\mathrm{TMS}} & =\nu_{\mathrm{FSR}}\left[\frac{m}{\pi} \cos ^{-1} \sqrt{g_{1 x} g_{2 x}}+\frac{n}{\pi} \cos ^{-1} \sqrt{g_{1 y} g_{2 y}}\right], \\
& =m \nu_{\mathrm{TMS}, x}+n \nu_{\mathrm{TMS}, y},
\end{aligned}
$$

where $g_{i x}=\left(1-L / R_{i x}\right), g_{i y}=\left(1-L / R_{i y}\right)$, and $R_{i x}$, $R_{i y}$ represent the radius of curvature of the $i$ th mirror of the cavity in the $x$ and $y$ directions, respectively.

With this definition, the direct measurement of the TMS can then be used to estimate the cavity $g$-factor.

\section{A. Measurement Technique}

The technique presented in this article determines the FSR and the TMS of a cavity from the resonances appearing in transmission as the laser frequency is scanned. Two lasers are used for the measurement: the first, serving as a master laser, is set to resonate in the cavity in its $\mathrm{TEM}_{00}$ mode; the second, phaselocked to the first, is held at an arbitrary offset frequency set by a local oscillator (LO) in the loop [17]. The phase-locked loop (PLL) ensures that the relative frequency of the two lasers remains constant.

At first, the optical cavity under test is locked to the fundamental mode of the master laser by using the Pound-Drever-Hall (PDH) technique [18]. The 
slave beam, after being appropriately mode matched, is injected into the cavity together with the main beam. At the cavity transmission, the two beams interfere, producing a beat note at their differential frequency as set by the PLL's LO frequency. Finally, the cavity resonance profile is measured by tracking the beat note's amplitude as a function of the LO frequency.

\section{B. Cavity Absolute Length Measurement}

As the frequency of the PLL's LO is swept, a resonance peak appears in transmission every time the relative frequency of the slave laser reaches a multiple of the cavity FSR. The FSR is then estimated by a least-squares fit of this set of measured resonant frequencies $\nu_{n}$ by the linear function $\nu_{n}=n \times \nu_{\mathrm{FSR}}$, where $n$ is the resonance's order.

\section{Cavity $g$-factor Measurement}

Following the FSR measurement, the TMS is measured by coupling the laser's fundamental mode into both the $\mathrm{TEM}_{00}$ cavity mode and the $\mathrm{TEM}_{01}$ or $\mathrm{TEM}_{10}$ spatial modes. This is obtained by introducing a small misalignment between the input beam and the cavity axis [19, Section II]. In particular, the coupling into the cavity's TEM $_{10}$ mode is obtained by a tilt of the cavity axis in yaw; a coupling into the cavity's TEM $_{01}$ mode is obtained by a tilt in pitch.

The measurement begins with the relative frequency of the two lasers set to a resonant frequency $\nu_{n}$ equal to an integer multiple $n$ of the cavity FSR: $\nu_{n}=n \times \nu_{\mathrm{FSR}}$. Then, the frequency of the auxiliary laser is scanned, while the cavity is held locked to the main laser. As their relative frequency equals the cavity's TMS, the auxiliary laser resonates in the cavity's $\mathrm{TEM}_{01}$ (or $\mathrm{TEM}_{10}$ ) mode and is transmitted by the cavity. The interference between the main beam's TEM $_{00}$ mode and the auxiliary beam's $\mathrm{TEM}_{01}\left(\right.$ or $\left.\mathrm{TEM}_{10}\right)$ mode produces a beat note on a photodetector (PD) at a frequency $\nu=\nu_{n}+\nu_{\mathrm{TMS}}$. The TMS is then measured by the frequency of the corresponding peak.

Both the TEM $\mathrm{T}_{01}$ and $\mathrm{TEM}_{10}$ modes have antisymmetric phases around the beam axis. As a result, their interference with the $\mathrm{TEM}_{00}$ mode has zero net power when integrated over the PD's transverse plane. To detect the beat note, it is necessary to break the symmetry of the beam spot just before the PD. This was done by partially clipping the beam with a razor blade in front of the $\mathrm{PD}$ but could also be done with a broadband quadrant PD.

\section{Characterization of the Arm Cavities of a Gravitational-Wave Interferometer}

This technique was tested on the arm cavities of the Caltech $40 \mathrm{~m}$ prototype, a testbed facility for the Laser Interferometer Gravitational-wave Observatory (LIGO) [20]. For the experiment, the interferometer was set in a dual-recycled Fabry-Perot Michelson configuration. In this setup, the two ( $40 \mathrm{~m}$ long) Fabry-Perot cavities, are connected in a Michelson configuration. Similar to the Advanced LIGO interferometers [21], the beam splitter's symmetric and antisymmetric outputs are coupled to the so-called recycling cavities: the power recycling cavity (PRC) at the symmetric port and the signal recycling cavity (SRC) at the antisymmetric port. All the cavity mirrors are suspended and hang on wires as simple pendulums, for seismic noise isolation.

Figure 1 shows the optical layout of this experiment. The main beam illuminating the interferometer was provided by a nonplanar-ring-oscillator laser (NPRO), amplified by a master oscillator power amplifier (MOPA) up to about $2 \mathrm{~W}$ at the time of the experiment [22]. Dedicated frequency and power prestabilization subsystems were enabled on the laser. After these stages, phase-modulation sidebands were added to the beam by electro-optic modulators

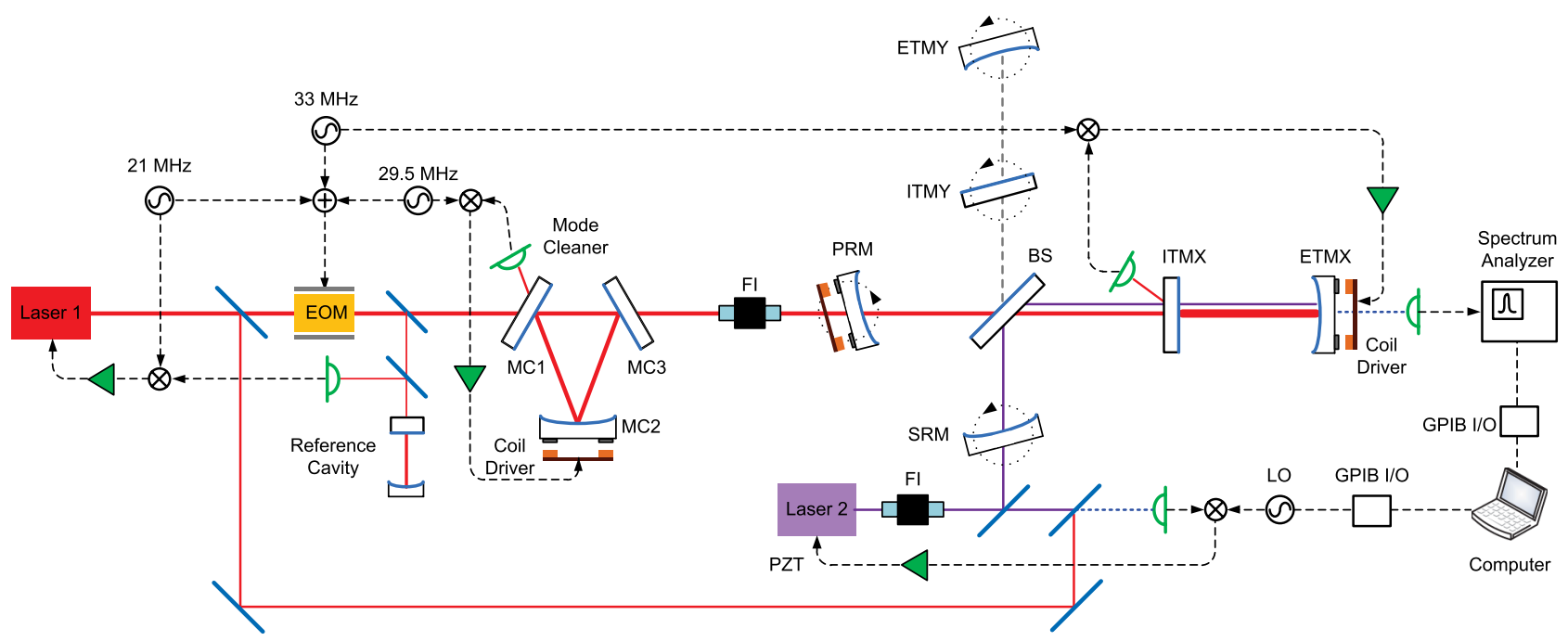

Fig. 1. (Color online) Interferometer setup for the $X$ arm measurement. While the arm to be measured is locked to laser 1 (main) by PDH locking, the rest of the interferometer is held misaligned. Laser 2 is phase-locked to laser 1 , and it is then injected through the signal recycling mirror (SRM). A beat note is detected in transmission by a spectrum analyzer. Its amplitude is recorded as the PLL's LO frequency and swept through several FSRs. 
(EOMs) for cavity locking. Finally, prior to entering the interferometer, the laser beam passed through a $13 \mathrm{~m}$-long triangular mode-cleaning cavity. The PM sideband frequencies are chosen to coincide with one of the FSRs of the mode-cleaning cavity.

The auxiliary beam was injected into the antisymmetric port of the interferometer, through the SRC's mirror (SRM). This solution allowed the beam to bypass the input mode-cleaner cavity.

The auxiliary beam was provided by a $700 \mathrm{~mW}$ NPRO, located outside the interferometer's vacuum envelope, on the same optics table hosting the dark port's optical setup. A simple flipper mirror was then used to enable the auxiliary beam to enter the interferometer at the time of the measurement.

A phase-lock optical system was set up on the main laser table by interfering pick-off beams from the two lasers on an RF photodiode (Thorlabs PDA255 or New Focus 1611). The PLL ensured that the auxiliary laser's frequency followed that of the main laser with an arbitrary tunable offset, by suppressing the relative phase fluctuations between the two lasers.

\section{Measurement Results}

The FSR and TMS were measured separately. In both measurements, the two laser beams were resonated only in the cavity to be examined by misaligning the other parts of the interferometer. The cavity was then locked to the main laser by the PDH technique [18].

A computer controlled the PLL's LO via a GPIB I/O interface. A script swept the LO frequency and monitored a spectrum analyzer connected to the PD in transmission. The power of the PD signal at the LO frequency was recorded at each step of the frequency scan.

\section{A. Arm Length Measurement}

The arm cavity to be measured was first locked to the main laser by controlling the end mirror with electromagnetic actuators. Then the auxiliary laser was injected into the interferometer. The PLL frequency was scanned by $\pm 20 \mathrm{MHz}$, a range corresponding to \pm 5 cavity FSRs, by sweeping the auxiliary laser's frequency first below and then above the main laser's frequency. The data obtained recording the power of the PD signal versus the PLL frequency was plotted as in Fig. 2. Resonance peaks were observed at multiples of the cavity FSR. The frequency $f_{0}$ of each peak was determined by a nonlinear least-squares fit of the $\mathrm{PD}$ signal amplitude $V_{\mathrm{PD}}$ with the function

$$
P_{\mathrm{PD}}(f)=\frac{P_{0}}{\sqrt{1+\left(f-f_{0}\right)^{2} / f_{c}^{2}}}+P_{\mathrm{off}}
$$

where $P_{0}, P_{\text {off }}$, and $f_{c}$ are additional fit parameters.

By fitting 10 resonances of the $X$ arm and five resonances of the $Y$ arm, $f_{0}$ was estimated with an error of $\sim 6-7 \mathrm{~Hz}$ and $\sim 9-11 \mathrm{~Hz}$, respectively. The residuals from the fitting show that these errors are statistical and not systematic.

The cavity FSR was estimated by a linear leastsquares fit of these resonant frequencies (right plot of Fig. 2). Table 1 shows the result of the fitting, as well as the derived values of the cavity length. The FSRs were estimated with statistical standard errors of $0.6 \mathrm{~Hz}$ and $3 \mathrm{~Hz}$ in the $X$ and $Y$ arms, respectively. Accordingly, the cavity lengths were determined with a precision of 6 and $30 \mu \mathrm{m}$, over an absolute length of $\sim 38.5 \mathrm{~m}$. However, the residuals in the linear fitting show deviations that are likely due to causes other than statistical fluctuations. In fact, deviations were observed at the level of $\sim 30-40 \mathrm{~Hz}$, equivalent to a displacement of $\sim 0.3-0.4 \mathrm{~mm}$ (see plotted residuals in Fig. 2). These fluctuations may be due to drift of the cavity alignment or actual length changes during the course of the measurement due to the thermal expansion of the concrete slab.

\section{B. Arm Cavity $g$-factor}

The arm cavity $g$-factor was measured by introducing a small misalignment in the input beam's axis, first in pitch, then in yaw in order to detect the resonances of the $\mathrm{TEM}_{01}$ mode and the $\mathrm{TEM}_{10}$ mode, respectively. The measurement started by locking the
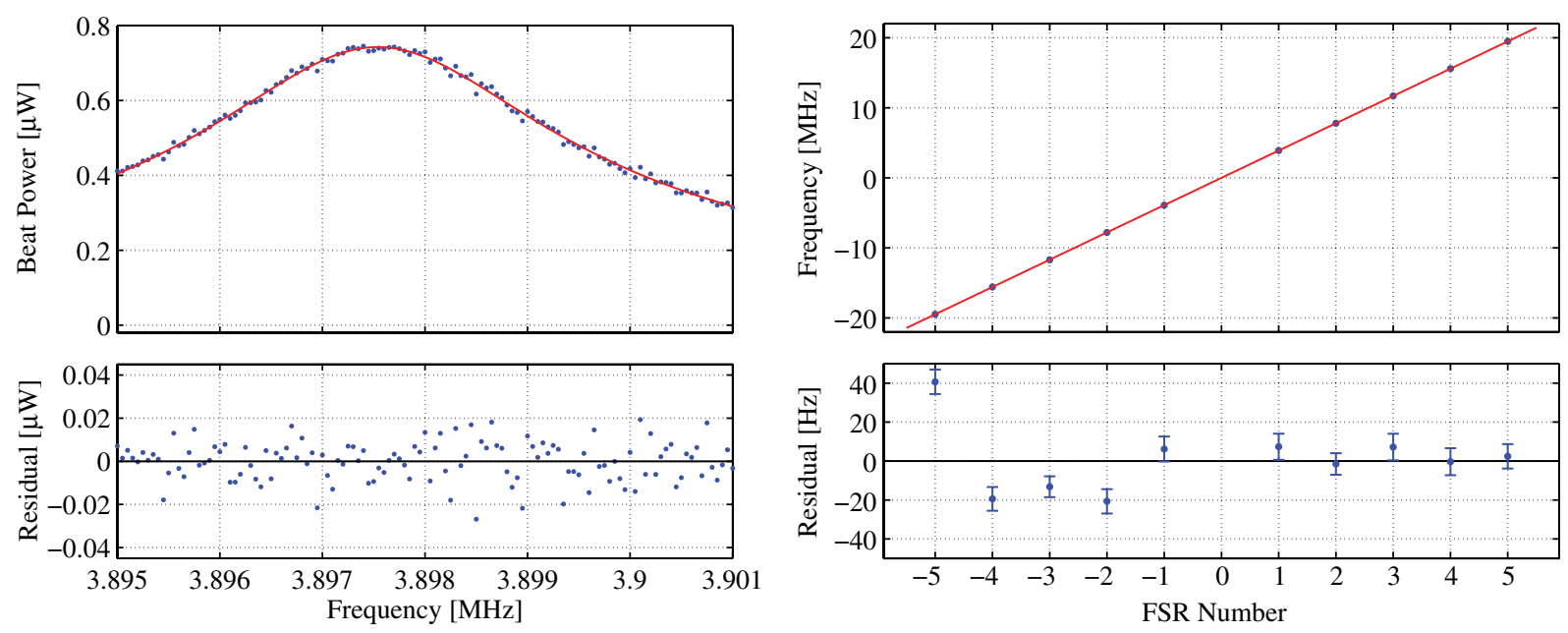

Fig. 2. (Color online) Laser frequency scan of a cavity resonance (left) and linear fit of 10 resonant frequencies (right). 
Table 1. Summary of Measurements on the Arm Cavities and on the PRC

\begin{tabular}{lc}
\hline \multicolumn{1}{c}{ Measured } \\
\hline \multicolumn{1}{c}{ arm } \\
$\nu_{\mathrm{FSR}}$ & $3897627.5 \pm 0.6 \mathrm{~Hz}$ \\
$\nu_{\mathrm{TMS}, H}$ & $1199048 \pm 20 \mathrm{~Hz}$ \\
$\nu_{\mathrm{TMS}, V}$ & $\boldsymbol{Y}$ arm \\
& $3873602 \pm 46 \mathrm{~Hz}$ \\
$\nu_{\mathrm{FSR}}$ & $1207790 \pm 23 \mathrm{~Hz}$ \\
$\nu_{\mathrm{TMS}, H}$ & $1189071 \pm 18 \mathrm{~Hz}$ \\
$\nu_{\mathrm{TMS}, V}$ & Derived \\
& $X$ arm \\
$L$ & $38.458326 \pm 6 \times 10^{-6} \mathrm{~m}$ \\
$g_{H}$ & $0.322885 \pm 1.5 \times 10^{-5}$ \\
$g_{V}$ & $0.311965 \pm 3.5 \times 10^{-5}$ \\
& $\boldsymbol{Y}$ arm \\
$L$ & $38.64622 \pm 3 \times 10^{-5} \mathrm{~m}$ \\
$g_{H}$ & $0.312013 \pm 1.7 \times 10^{-5}$ \\
$g_{V}$ & $0.326144 \pm 1.4 \times 10^{-5}$ \\
$l_{\mathrm{PRC}}$ & PRC \\
Asymmetry & $2.217 \pm 2 \times 10^{-3} \mathrm{~m}$ \\
\hline
\end{tabular}

${ }^{a} \nu_{\mathrm{FSR}}, \nu_{\mathrm{TMS}, H}$, and $\nu_{\mathrm{TMS}, V}$ are the FSR and the TMS for the horizontal mode and the vertical mode, respectively. $L$ is the cavity length. $g_{H}$ and $g_{V}$ are the $g$-factors for the horizontal and vertical modes. $l_{\mathrm{PRC}}$ and Asymmetry are the length of the PRC and the difference of the Michelson arm lengths.

cavity to the main laser's fundamental mode. The amplitude of the PD signal was recorded and plotted against the PLL frequency.

The frequency scan revealed additional resonance peaks due to higher-order modes (Fig. 3). The first peak was produced by the $\mathrm{TEM}_{00}$ mode resonance; the second corresponded to the spatial mode resonance. The TMS was determined by measuring the frequencies of the $\mathrm{TEM}_{10}$ and $\mathrm{TEM}_{01}$ modes and then comparing them with the frequency of the $\mathrm{TEM}_{00}$ mode.

The $\mathrm{TEM}_{10}$ (or $\mathrm{TEM}_{01}$ ) peak was identified by matching it with its expected location as calculated from the mirror's nominal radius of curvature. The fitting of the $\mathrm{TEM}_{10}$ and $\mathrm{TEM}_{01}$ resonances allowed us to estimate $f_{0}$ with errors of 15 and $46 \mathrm{~Hz}$. The fitting residuals indicate that statistical fluctuations are responsible for these deviations.

It should be noted that the $g$-factor measurements presented here and the values obtained by direct measurements of the mirrors' radii of curvature do not agree. Phase-map measurements of the mirrors obtained by a Fizeau interferometer estimated the radii of curvature of the end and input mirrors of the $X$ and $Y$ arms to be 57.57, 57.68, 7280, and 7210 (all in meters), respectively. These numbers, combined with the measured cavity lengths, give us $g$ factors of $2 \sim 6 \%$ larger than the measured values. In general, the individual radii of curvature of mirrors in a two-mirror cavity cannot be directly derived from measured $g$-factors. However, we can still learn something about the mirror curvatures if we take into account that typically phase-map measurements of flat mirrors are more accurate than those
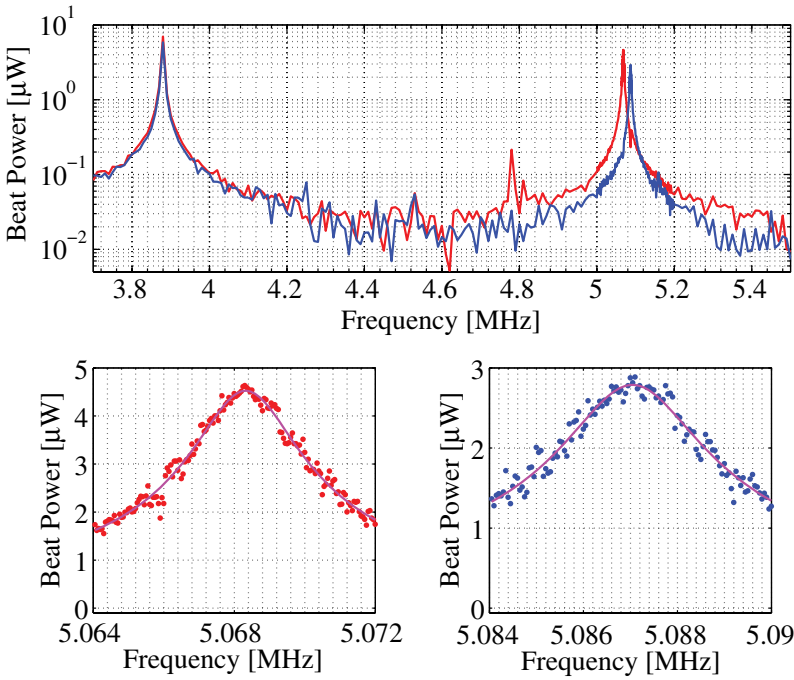

Fig. 3. (Color online) Measurement of TMS in the $Y$ arm. The curves are the results of the frequency scans for the horizontal and vertical modes, respectively. In the upper plot, the left peak is the cavity's fundamental resonance, while the two peaks on the right are the resonances of the spatial modes (zoomed-in in the lower plots). A fit of the data points in these peaks (continuous lines) is used to determine their frequencies.

of highly curved mirrors $[23,24]$. For instance, phase-map measurements of the nominally flat mirrors in use in our lab have estimated radii of curvature ranging between $-100 \mathrm{~km}$ and $+6 \mathrm{~km}$. Finite radii like these affect the $g$-factors by less than $0.6 \%$, compared to an ideally flat mirror. For this reason, most likely the observed discrepancies are due to the curvature of the end mirrors. Astigmatism in the end mirrors could explain the $\mathrm{TEM}_{01} / \mathrm{TEM}_{10}$ mode splitting and justify the difference in the $g$-factors by $3 \sim 4 \%$. In particular, by assuming perfectly flat input mirrors, our measurements could be explained by an astigmatism of $\sim 1 \mathrm{~m}$ over end mirrors with radii of curvature of $56 \sim 57 \mathrm{~m}$.

The two astigmatic modes were observed in the vertical and horizontal main axes in both arm cavities, rather than along arbitrary axes in each of them. This might occur if the astigmatism was related to the wedge angle on the cavity mirrors. Both the input and the output mirrors have wedge angles of $1^{\circ}$ and $2.5^{\circ}$, respectively, and they were both installed with the angles on the horizontal axis. This nondegeneracy of the cavity's $\mathrm{TEM}_{10} / \mathrm{TEM}_{01}$ resonances was also confirmed by an experiment in which the cavity was locked to either of these modes. By misaligning the cavity in pitch or yaw, it was possible to lock the cavity in either the $\mathrm{TEM}_{10}$ mode or the $\mathrm{TEM}_{01}$ mode, but never on both at the same time. This was evidence that the modes were indeed separated by much more than the cavity half linewidth of $1.6 \mathrm{kHz}$.

\section{Power Recycling Cavity (PRC)}

Determining the length of the other cavities, such as the recycling cavity, is also very important in order to achieve a stable control of the interferometer. For this reason, we tested this technique on the PRC. 
This cavity is formed by the power recycling mirror (PRM) at one end and at the other end by the Michelson "compound mirror," an effective mirror defined by the beam splitter and the input mirrors (ITM) of the arm cavities. The asymmetry in this short Michelson causes it to have a frequencydependent reflectivity [25].

For this measurement, the auxiliary beam was introduced through a misaligned SRM. The transmitted beam was detected at the reflection port of the interferometer using the output of the Faraday isolator. The Michelson and the PRC were locked to the main laser beam, by actuating on the beam splitter and the PRM. Because the PRC length is much shorter than the arms', the laser frequency had to be scanned by several hundreds of megahertz in order to explore one cavity FSR. This was easily accomplished thanks to the large dynamic range of the PLL, in contrast to previous length measurement techniques, which had been limited to only very long cavities with smaller FSRs.

The measured profile of the transmitted power showed a complex pattern, as expected from the frequency-dependent response of the Michelson (Fig. 4). The data were fit with an analytical model for the cavity transmissivity (details in Appendix A), obtaining an estimate of the cavity absolute length and of the Michelson differential length (asymmetry) with a precision of $2 \mathrm{~mm}$ and $3 \mathrm{~mm}$, respectively (see Table 1). The derived cavity length and Michelson asymmetry can be compared to the values set approximating at the time of design/construction of the interferometer: $l_{\text {prc }}($ design $)=2.257 \mathrm{~m} ; l_{\text {asy }}($ design $)=0.451 \mathrm{~m}$. The uncertainties on the PRC parameters were then derived from the statistical fits with the same method described above for the arm cavities.

\section{Measurement Precision}

The precision of the FSR and TMS measurements listed in Table 1 was determined by the statistical
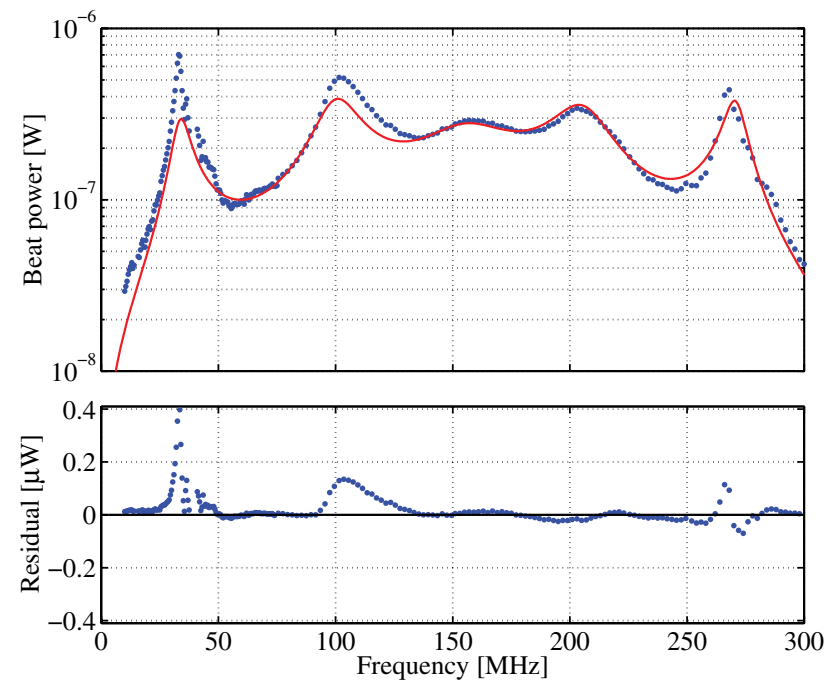

Fig. 4. (Color online) Frequency scan of the PRC: measured beat power (bold dots) and fitting curve (continuous line). errors in the parameters of the nonlinear leastsquares fit of the data. The difference between the two arms was due to a larger number of data points measured for the $X$ arm.

In principle, since the cavity is locked to the main laser, the best precision of the FSR and TMS measurements achievable by this technique is set by the laser frequency and intensity noise and by the noise in the PDH loop. In reality, fluctuations of the cavity stored power due to oscillations of the cavity alignment may affect the beat note's amplitude and thus increase the errors in the fit parameters. In our case, since the angular degrees of freedom of the cavities were uncontrolled, the mirrors' angular motion was likely the main cause of the measured fluctuations in the beat note signal.

In the future the technique could be greatly improved by measuring the phase of the beat note rather than its amplitude. The cavity resonance could then be measured with more accuracy and precision by identifying the frequency at which the beat note's phase flips by $180^{\circ}$. This would render the measurement intrinsically immune to unwanted fluctuations of the beam power. For instance, the phase could be detected by measuring the transfer function between the PLL's LO signal and the beat note.

\section{Conclusions}

We demonstrated a new interferometric technique to measure the FSR and the TMS of optical cavities of various lengths. It allowed measurements of the length and the $g$-factor of a Fabry-Perot cavity with a precision of $1 \mathrm{ppm}$ and $10 \mathrm{ppm}$, respectively.

Compared to previous methods, this technique can be applied to optical cavities of lengths ranging from a few meters to several kilometers. With our experiment we proved that the technique is compatible with a complex optical system, comprising coupled cavities. Not requiring structural modifications to the optical system on which it is applied, it provides a convenient option in circumstances in which an in situ and noninvasive measurement tool for cavity length and $g$-factor is needed.

\section{Appendix A: PRC Transmissivity}

When the primary laser is resonant, the transmissivity of the PRC can be written as a function of the PLL's LO frequency $\Delta \omega=\omega_{\mathrm{psl}}-\omega_{\text {aux }}$ in the following way:

$$
T_{\mathrm{prc}}=\left|\frac{t_{\mathrm{prm}} r_{\mathrm{itm}} e^{-i 2 \Delta \omega l_{\mathrm{prc}} / c} \sin \left(\Delta \omega l_{\mathrm{asy}} / c\right)}{1+r_{\mathrm{prm}} r_{\mathrm{itm}} e^{-i 2 \Delta \omega l_{\mathrm{prc}} / c} \cos \left(\Delta \omega l_{\mathrm{asy}} / c\right)}\right|^{2},
$$

where $t_{\text {prm }}$ is the PRM transmittance, $r_{\text {itm }}$ is the ITM reflectance, $l_{\text {asy }}$ is the asymmetry (i.e., the Michelson's differential length), and $l_{\text {prc }}$ is the effective length of the PRC defined as $\left.l_{\text {prc }} \equiv l_{\text {prm-bs }}+\left(l_{x}+l_{y}\right) / 2\right)$, where $l_{\text {prm-bs }}$ is the distance between the beam splitter (BS) and the PRM, and $l_{x}$ and $l_{y}$ are the lengths of the Michelson arms. The amplitude of the beat note in transmission is then proportional to $\sqrt{T_{\mathrm{prc}}}$. The 
parameters $l_{\text {prc }}$ and $l_{\text {asy }}$ determine $T_{\text {prc }}(\Delta \omega)$ univocally: a given function $T_{\mathrm{prc}}(\Delta \omega)$ can be produced by only one couple of parameters $\left(l_{\text {prc }}, l_{\text {asy }}\right)$.

We thank Yoichi Aso, Robert Ward, and Hiro Yamamoto for illuminating discussions on this technique. We also thank the rest of the $40 \mathrm{~m}$ team for building and maintaining the interferometer during the course of this work. The LIGO Observatories were constructed by the California Institute of Technology and the Massachusetts Institute of Technology with funding from the National Science Foundation under cooperative agreement PHY-0757058. This paper has been assigned a LIGO Document Number of P1200048.

\section{References}

1. A. Araya, S. Telada, K. Tochikubo, S. Taniguchi, R. Takahashi, K. Kawabe, D. Tatsumi, T. Yamazaki, S. Kawamura, S. Miyoki, S. Moriwaki, M. Musha, S. Nagano, M.-K. Fujimoto, K. Horikoshi, N. Mio, Y. Naito, A. Takamori, and K. Yamamoto, "Absolute-length determination of a long-baseline FabryPerot cavity by means of resonating modulation sidebands," Appl. Opt. 38, 2848-2856 (1999).

2. M. Rakhmanov, F. Bondu, O. Debieu, and R. L. Savage, Jr., "Characterization of the LIGO $4 \mathrm{~km}$ Fabry-Perot cavities via their high-frequency dynamic responses to length and laser frequency variations," Class. Quantum Grav. 21, S487-S492 (2004).

3. B. P. Abbott and The LIGO Scientific Collaboration, "LIGO: The Laser Interferometer Gravitational-wave Observatory," Rept. Prog. Phys. 72, 076901 (2009).

4. T. Accadia and The Virgo Collaboration, "Status and perspectives of the Virgo gravitational wave detector," J. Phys.: Conf. Ser. 23, 012074 (2010).

5. K. Kuroda, "Status of LCGT," Classical Quantum Gravity 27, 084004 (2010).

6. H. Grote and The GEO Collaboration, "The GEO 600 status," Class. Quantum Grav. 27, 084003 (2010).

7. D. Redding, M. Regehr, and L. Sievers, "Dynamic models of Fabry-Perot interferometers," Appl. Opt. 41, 2894-2906 (2002).

8. R. L. Ward, R. Adhikari, B. Abbott, R. Abbott, D. Barron, R. Bork, T. Fricke, V. Frolov, J. Heefner, A. Ivanov, O. Miyakawa, K. McKenzie, B. Slagmolen, M. Smith, R. Taylor, S. Vass, S. Waldman, and A. Weinstein, "DC readout experiment at the Caltech $40 \mathrm{~m}$ prototype interferometer," Class. Quantum Grav. 25, 114030 (2008).
9. R. L. Ward, "Length sensing and control of a prototype advanced interferometric gravitational wave detector," Ph.D. thesis (California Institute of Technology, 2010).

10. A. Stochino, "Design and characterization of optical cavities and length sensing and control system of an advanced gravitational wave interferometer," Ph.D. thesis (University of Siena, 2010). LIGO Document P1000148, available at https://dcc.ligo.org.

11. J. Ye, "Absolute measurement of a long, arbitrary distance to less than an optical fringe," Opt. Lett. 29, 1153-1155 (2004).

12. M. Rakhmanov, M. Evans, and H. Yamamoto, "An optical vernier technique for in situ measurement of the length of long Fabry-Perot cavities," Meas. Sci. Technol. 10, 190-194 (1999).

13. N. Uehara and K. Ueda, "Accurate measurement of the radius of curvature of a concave mirror and the power dependence in a high-finesse Fabry-Perot interferometer," Appl. Opt. 34, 5611-5619 (1995).

14. R. DeVoe and R. Brewer, "Laser-frequency division and stabilization," Phys. Rev. A 30, 2827-2829 (1984).

15. A. Siegman, Lasers (University Science Books, 1986).

16. S. Habraken and G. Nienhuis, "Modes of a twisted optical cavity," Phys. Rev. A 75, 033819 (2007).

17. J. B. Armor, Jr. and S. R. Robinson, "Phasealock control considerations for coherently combined lasers," Appl. Opt. 18, 3165-3175 (1979).

18. R. W. P. Drever, J. L. Hall, F. V. Kowalski, J. Hough, G. M. Ford, A. J. Munley, and H. Ward, "Laser phase and frequency stabilization using an optical resonator," Appl. Phys. B: Lasers Opt. 31, 97-105 (1983).

19. D. Z. Anderson, "Alignment of resonant optical cavities," Appl. Opt. 23, 2944-2949 (1984).

20. A. Weinstein, "Advanced LIGO optical configuration and prototyping effort," Class. Quantum Grav. 19, 1575-1584 (2002).

21. G. M. Harry and The LIGO Scientific Collaboration, "Advanced LIGO: the next generation of gravitational wave detectors," Class. Quantum Grav. 27, 084006 (2010).

22. B. Willke, K. Danzmann, M. Frede, P. King, D. Kracht, P. Kwee, O. Puncken, R. L. Savage, Jr., B. Schulz, F. Seifert, C. Veltkamp, S. Wagner, P. Weels, and L. Winkelmann, "Stabilized lasers for advanced gravitational wave detectors," Class. Quantum Grav. 25, 114040 (2008).

23. K.-E. Elssner, A. Vogel, J. Grzanna, and G. Schulz, "Establishing a flatness standard," Appl. Opt. 33, 2437-2446 (1994).

24. K.-E. Elssner, R. Burow, J. Grzanna, and R. Spolaczyk, "Absolute sphericity measurement," Appl. Opt. 28, 4649-4661 (1989).

25. D. Sigg, N. Mavalvala, J. Giaime, P. Fritschel, and D. Shoemaker, "Signal extraction in a power-recycled Michelson interferometer with Fabry-Perot arm cavities by use of a multiple-carrier frontal modulation scheme," Appl. Opt. 37, 5687-5693 (1998). 